\title{
Right hemidiaphragmatic paralysis after cervical transforaminal epidural steroid injection: illustrative case
}

\author{
Molly Farrell, BS, ${ }^{1}$ Ezek Mathew, BS, ${ }^{1}$ Martin Weiss, DO, ${ }^{2}$ and Rob Dickerman, DO, PhD ${ }^{3}$ \\ ${ }^{1}$ Texas College of Osteopathic Medicine, Fort Worth, Texas; ${ }^{2}$ Cardiology Department, Baylor Scott \& White Medical Center, McKinney, Texas; and ${ }^{3}$ Department of Neurosurgery, \\ Presbyterian Hospital, Plano, Texas
}

\begin{abstract}
BACKGROUND Cervical radiculopathy is a common cause of neck pain, with radiation into the upper extremity in a dermatomal pattern. Corticosteroid injection is a conservative management option with a low risk of major adverse events. No reviewed literature or case reports have implicated phrenic nerve injury secondary to cervical transforaminal epidural steroid injection (CTFESI).

OBSERVATIONS A 45-year-old man with severe right C6 radiculopathy secondary to a large right-sided C5-6 herniated intervertebral disc presented to the pain management clinic, where he received a right-sided C6 CTFESI. An hour after injection, the patient experienced shortness of breath, which was found to be caused by right diaphragmatic paralysis. The patient underwent a C5-6 anterior cervical discectomy and fusion, which provided complete relief of his radicular symptoms. However, the right hemidiaphragmatic paralysis remained at the 1-year postoperative visit.

LESSONS Thorough literature review showed no established explanations for phrenic nerve injury after CTFESI. In this study, the authors explored the suspected mechanisms of possible injury to the phrenic nerve. Epidural corticosteroid injection is considered to be a safe option for conservative management of cervical radiculopathy. This study unveiled a unique and important adverse event that should be considered before a patient receives CTFESI.
\end{abstract}

https://thejns.org/doi/abs/10.3171/CASE20113

KEYWORDS hemidiaphragmatic paralysis; cervical transforaminal epidural steroid injection; CTFESI

Cervical radiculopathy is a common cause of neck pain characterized by radiation of paresthesia, pain, and/or weakness into the upper extremity. A retrospective, population-based study in Rochester, Minnesota, found cervical radiculopathy to be a common condition with an age-adjusted incidence of 83.2 per 100,000 persons per year. ${ }^{1}$ This condition has a peak incidence of 202.9 per 100,000 persons per year in individuals 50-54 years old. ${ }^{1}$ Vertebral spondylosis, cervical intervertebral disc herniation, and spondylolisthesis frequently cause nerve impingement, most commonly of the $\mathrm{C} 7$ or $\mathrm{C} 6$ nerve roots. ${ }^{1,2}$ Conservative management options include immobilization, traction, physical therapy, manipulative medicine, analgesic medications, and corticosteroid injections. ${ }^{2}$

There are two approaches for corticosteroid injections, interlaminar and transforaminal, each of which has a low risk of major adverse events. ${ }^{3}$ Adverse events could include epidural hematoma, infection, allergic reaction, seizure, nerve damage, or intravascular injection. ${ }^{4}$ No reviewed literature or case reports have indicated hemidiaphragmatic paralysis secondary to epidural steroid injections. In this study, we explore the suspected mechanisms of possible injury to the phrenic nerve. Etiologies to consider include the particulate effects of steroids, technique of injection, unique anatomical variations, and neuralgic amyotrophy (NA).

\section{Illustrative Case}

A 45-year-old man with no significant medical history presented to our clinic with severe right $\mathrm{C} 6$ radiculopathy. Magnetic resonance imaging showed a large right-sided C5-6 herniated disc. To relieve pain, the patient received a right-sided transforaminal C5-6 epidural steroid injection with propofol sedation. The cervical transforaminal epidural steroid injection (CTFESI) was performed with the patient

ABBREVIATIONS APN = accessory phrenic nerve; $C T$ = computed tomography; CTFESI = cervical transforaminal epidural steroid injection; IgG = immunoglobulin G; $N A=$ neuralgic amyotrophy.

INCLUDE WHEN CITING Published May 10, 2021; DOI: 10.3171/CASE20113.

SUBMITTED November 13, 2020. ACCEPTED February 15, 2021.

(C) 2021 The authors, CC BY-NC-ND 4.0 (http://creativecommons.org/licenses/by-nc-nd/4.0/). 
in the lateral decubitus position. After confirming the correct oblique view of the $\mathrm{C} 6$ foramen, a needle was passed into the neck through a point overlying the posterior half of the C6 foramen. Under direct, real-time fluoroscopy view, a small volume of contrast was injected. Upon good visualization of the C6 nerve, a 1-mL mixture of $40 \mathrm{mg}$ Kenalog (triamcinolone acetonide) and $1 \%$ lidocaine was injected. Approximately 1 hour after the injection as the patient was riding home, he felt short of breath. The patient, a cardiologist, performed a sniff test the next day at work, which led to the discovery of hemidiaphragmatic paralysis.

Because the hemidiaphragmatic paralysis and radicular pain persisted, the patient presented to the neurosurgery clinic for assessment. Preoperative chest radiographs showed evidence of atelectasis of the right middle and right lower lobes, consistent with hemidiaphragmatic paralysis of the right side. A subsequent sniff test before surgery confirmed right hemidiaphragmatic paralysis. Six weeks later, the decision was made by the patient and his healthcare provider to undergo a C5-6 anterior cervical discectomy and fusion. There was a complete resolution of the radicular symptoms postoperatively; however, the diaphragmatic paralysis persisted, even at the 1-year postoperative visit.

\section{Discussion}

\section{Observations}

No current studies have reported hemidiaphragmatic paralysis secondary to CTFESI, making this case unique. Reports of major adverse events, such as quadriplegia, stroke, and death, after CTFESI do exist. ${ }^{4}$ However, these events are believed to stem from brain or spinal cord infarction from intravascular injection of corticosteroids. $^{4-8}$ In those cases, the neurological deficit is sometimes permanent, often involving the central nervous system more than peripheral nerves. ${ }^{9}$ Similarly, in this case, the adverse event was significant and permanent.

\section{Lessons}

The goal of the CTFESI technique is to provide placement of corticosteroids in direct proximity to an inflamed nerve root. ${ }^{4,5}$ The corticosteroid then suppresses the physiological mechanism of inflammation. In terms of particle size, corticosteroids can be divided into nonparticulate and particulate steroids. Particulate steroids may contain particles that are larger than $1000 \mu \mathrm{m}$ in some cases. The significance of size rests on the potential of these particulates to aggregate, which can cause blockage of vasculature or lead to microemboli. ${ }^{10}$ This possibility was examined in a mouse model, in which injection of particulate steroids did lead to occlusion of vasculature. This effect predominated in smaller vessels and occurred as quickly as 5 minutes after injection in some cases. ${ }^{11}$ With this background, although inadvertent intraarterial injection of particulate corticosteroid seems unlikely, procedural access to the posterior inferior neuroforamen does place the needle within close proximity to vital vasculature in the cervical region. Although particulate corticosteroids have delayed, sustained effects when used in treatment, the time course of embolic side effects in humans is not well defined. Ischemia and infarction to neural tissue induced by intraarterial injection of particulate corticosteroids may parallel the time course observed with the hemidiaphragmatic paralysis in this case. $^{4,5}$ Because Kenalog, a particulate steroid, was used during the procedure, it is possible that this neurological dysfunction had a vascular origin.
It must be noted that particulate steroid-induced microvasculature occlusion and subsequent ischemia do not fully explain the isolated symptom of hemidiaphragmatic paralysis. This explanation requires that embolism or ischemia occur selectively to the arteries that supply the phrenic nerve while sparing supply of the cervical cord. The vascular supply to the phrenic nerve is not fully documented, and anatomical variations do exist, making this distinction difficult to elucidate. However, the use of particulate steroids and evidence of adverse neurological events in other foraminal injections are concerning. There are no reports of such complications with nonparticulate corticosteroids, perhaps making them a safer alternative if diaphragmatic paralysis is indeed secondary to an embolic effect. This finding culminated in a recommendation to use nonparticulate steroids for lumbar transforaminal injections. ${ }^{12}$ In light of the major adverse event in this case and the deleterious neurological effects of particulate steroids in other cases, it may be wise to instead use nonparticulate steroids for CTFESI.

According to Seddon, the three main types of nerve injury are neurotmesis, axonotmesis, and neurapraxia. ${ }^{13}$ The most severe of these types is neurotmesis, which typically arises when a nerve is physically transected. Phrenic nerve neurotmesis is a complication that can arise after thoracic surgery or as a result of trauma. ${ }^{14,15}$ Axonotmesis is less severe but involves damage of the myelin sheath, which degrades the continuity of nerve conduction. Although various causes exist for axonotmesis, similar to neurotmesis, the deficit is often irreversible. The least severe and most reversible type is neurapraxia. A mechanical cause of neurapraxia is traction or abnormal positioning. Considering that the neck may be rotated, flexed, or extended during CTFESI, the traction force imposed on the phrenic nerve could have contributed to the pathology of our patient. ${ }^{16}$

A possible explanation for this phenomenon of right hemidiaphragmatic paralysis is Parsonage-Turner syndrome, also known as NA. Classically, a patient experiences severe pain in the shoulder area, although the exact distribution can vary. After a variable period of 1 to 28 days, motor weakness may occur in the arm or shoulder area, typically within the C5-7 distribution; sensory deficits may occur in some cases. ${ }^{17,18}$ Although this condition favors the lower parts of the brachial plexus, the lumbosacral plexus can be involved in addition to other nerves. ${ }^{19,20}$ Phrenic nerve involvement occurs in an estimated $5 \%$ to $10 \%$ of patients. ${ }^{21}$ Nerve conduction studies may show patchy transmission or increase response latency of affected nerves; in later disease progression, the nerve conduction capacity of the phrenic nerve may be nonexistent, as happened with our patient. ${ }^{18}$ As in our patient's case, multiple case reports detail the existence of atelectasis observed on chest computed tomography (CT) concurrent with the diaphragmatic paralysis. ${ }^{22}$ Ultimately, neurological deficit of the brachial plexus, akin to cervical radiculopathy, with progressive symptoms unresolved by surgical intervention may point to $\mathrm{NA}$.

If the diagnosis is NA, it necessitates an explanation of how the condition occurred. Currently, the literature is divided regarding the cause, citing factors that range from genetic predisposition to infectious triggers and mechanical predisposition to injury. ${ }^{20,21}$ Although most cases are considered idiopathic, a few cases of NA inherited in an autosomal-dominant fashion have been noted. ${ }^{21}$ More research implicates the impact of SEPT9 gene mutations at the $17 q 25$ locus. $^{18,19}$ Other possible causes of NA include complications due to viral infections, as detailed in a two-case report. One 
patient was diagnosed with diverticulitis and developed suddenonset left hemidiaphragmatic paralysis within days. Viral serology results demonstrated elevated cytomegalovirus immunoglobulin $G$ (lgG) levels as well as varicella zoster $\lg G$ and Epstein-Barr lgG. Another patient presented with right hemidiaphragmatic paralysis that developed within 3 months after herpes zoster affected his T7-8 dermatome. ${ }^{23}$ The evidence for mechanical trauma is rather nondescript, but it does seem to involve the affected shoulder or the general nerve distribution of the brachial plexus. ${ }^{23,24}$ Perhaps for the patient, the right-sided mild foraminal stenosis at the C3-4 level seen on CT served as a possible structural cause because the $\mathrm{C} 4$ nerve root is heavily involved in diaphragmatic innervation. A prior infection that predisposed the patient to developing this condition may have been involved.

Although the exact cause has not been isolated, most sources elaborate on a common conception that an autoimmune reaction is generated, leading to variable degeneration of affected nerves over time. $^{17}$ As the body of knowledge grows for NA, it may become easier to identify the common genetic, structural, and immunological factors that contribute to this condition. However, the occurrence of hemidiaphragmatic paralysis immediately after CTFESI makes NA a less likely cause because NA appears to develop over a longer period. Regarding the estimated probability of recovery, in a series of 99 patients, $75 \%$ to $90 \%$ of patients with NA without diaphragmatic involvement recovered normal strength by 3 years; this finding was not quantified by examination of nerve conduction. However, the involvement of diaphragmatic paralysis was associated with a decreased rate of recovery because three of the four patients with diaphragmatic paralysis still had evidence of an immobile hemidiaphragm 2.5 to 4 years after NA. ${ }^{20}$ Other sources have described varying rates of recovery, but because of the rarity of hemidiaphragmatic paralysis, an estimated percentage of recovery could not be quantified. Unfortunately, it has been noted that the recovery of patients with NA that manifests with hemidiaphragmatic paralysis is markedly reduced in comparison to that of patients with NA without diaphragmatic involvement. ${ }^{17,20,25}$ Although NA is less likely to be present, the propensity for diaphragmatic paralysis associated with this pathology may be important to note.

The phrenic nerve classically arises from the C4 nerve root with contributions of the $\mathrm{C} 3$ and $\mathrm{C} 5$ nerve roots and allows for breathing by supplying the ipsilateral diaphragmatic musculature. ${ }^{26}$ Because of the circumstances surrounding hemidiaphragmatic paralysis, direct needle injury may have been a cause of phrenic nerve damage in our patient. The phrenic nerve, as it passes laterally in the cervical spine and in the craniocaudal direction, could have easily been injured or transected by the needle, especially with the transforaminal approach. Additionally, the presence of an accessory phrenic nerve (APN) is common and has been shown in $36.5 \%$ to $61.8 \%$ of phrenic nerves with variable origin, course, and communication with the respective phrenic nerve. ${ }^{27,28}$ The most common origin of an APN was the ansa cervicalis, which arises from $\mathrm{C} 3$ and $\mathrm{C} 4$, or the nerve to the subclavius, which arises from $\mathrm{C} 5$ and $\mathrm{C} 6 .{ }^{27,28}$ Because of an unforeseen anatomical variation, direct needle injury to the phrenic nerve may cause permanent hemidiaphragmatic paralysis. Therefore, phrenic nerve injury is an important consideration for cervical surgery, manipulation, trauma, brachial plexus nerve block, and cardiothoracic surgery.

The purpose of this study is to examine the hemidiaphragmatic paralysis that occurred after routine corticosteroid injection. This neurological deficit could be due to physical transection of the phrenic nerve or any of the factors that lead to NA. Under the category of neurapraxia, patient positioning is the most likely factor that can impact phrenic paralysis. Ultimately, the most likely cause of this neurological dysfunction is the use of particulate steroids, which may trigger an embolic or occlusive event in the vasculature supplying the phrenic nerve. This case necessitates extensive review of the adverse effects of particulate steroids in CTFESI and other neurological procedures in which microvasculature may be difficult to visualize.

\section{References}

1. Radhakrishnan K, Litchy WJ, O'Fallon WM, Kurland LT. Epidemiology of cervical radiculopathy. A population-based study from Rochester, Minnesota, 1976 through 1990. Brain. 1994;117(pt 2):325-335.

2. lyer S, Kim HJ. Cervical radiculopathy. Curr Rev Musculoskelet Med. 2016;9(3):272-280.

3. El-Yahchouchi CA, Plastaras CT, Maus TP, et al. Adverse event rates associated with transforaminal and interlaminar epidural steroid injections: a multi-institutional study. Pain Med. 2016;17(2): 239-249.

4. Schneider BJ, Maybin S, Sturos E. Safety and complications of cervical epidural steroid injections. Phys Med Rehabil Clin N Am. 2018;29(1):155-169.

5. Palmer WE. Spinal injections for pain management. Radiology. 2016;281(3):669-688.

6. Beckman WA, Mendez RJ, Paine GF, Mazzilli MA. Cerebellar herniation after cervical transforaminal epidural injection. Reg Anesth Pain Med. 2006;31(3):282-285.

7. Brouwers PJAM, Kottink EJBL, Simon MAM, Prevo RL. A cervical anterior spinal artery syndrome after diagnostic blockade of the right C6-nerve root. Pain. 2001;91(3):397-399.

8. Ludwig MA, Burns SP. Spinal cord infarction following cervical transforaminal epidural injection: a case report. Spine (Phila Pa 1976). 2005;30(10):E266-E268.

9. Epstein NE. The risks of epidural and transforaminal steroid injections in the spine: commentary and a comprehensive review of the literature. Surg Neurol Int. 2013;4(suppl 2):S74-S93.

10. Dietrich TJ, Sutter R, Froehlich JM, Pfirrmann CW. Particulate versus non-particulate steroids for lumbar transforaminal or interlaminar epidural steroid injections: an update. Skeletal Radiol. 2015;44(2):149 155.

11. Laemmel E, Segal N, Mirshahi M, et al. Deleterious effects of intraarterial administration of particulate steroids on microvascular perfusion in a mouse model. Radiology. 2016;279(3):731-740.

12. Schneider BJ, McCormick ZL, Smith CC. Particulate or nonparticulate steroids for lumbar transforaminal injections. Pain Med. 2017;18(9): 1817-1818.

13. Seddon HJ. Three types of nerve injury. Brain. 1943;66(4):237-288.

14. Whiteley J, Shoeib M, Bilancia R. latrogenic phrenic nerve palsy. Shanghai Chest. 2020. doi:10.21037/shc-2019-amp-01

15. Biso GMNR, Munakomi S. Neuroanatomy, neurapraxia. In: StatPearls. Internet. StatPearls Publishing; 2020. Accessed September 26, 2020. https://www.ncbi.nlm.nih.gov/books/NBK557746

16. Altafulla J, Yilmaz E, Lachkar S, et al. Neck movement during cervical transforaminal epidural injections and the position of the vertebral artery: an anatomical study. Acta Radiol Open. 2019;8(3): 2058460119834688.

17. Mulvey DA, Aquilina RJ, Elliott MW, et al. Diaphragmatic dysfunction in neuralgic amyotrophy: an electrophysiologic evaluation of 16 patients presenting with dyspnea. Am Rev Respir Dis. 1993;147(1):66-71.

18. Blanco-Aparicio M, Montero-Martínez C, Couto-Fernández D, et al. Unilateral painful diaphragm paralysis as the only sign of 
amyotrophic neuralgia. Article in Spanish. Arch Bronconeumol. 2010;46(7):390-392.

19. van Alfen $\mathrm{N}$. The neuralgic amyotrophy consultation. J Neurol. 2007;254(6):695-704.

20. Tsairis P, Dyck PJ, Mulder DW. Natural history of brachial plexus neuropathy. Report on 99 patients. Arch Neurol. 1972;27(2):109-117.

21. Kumar A, Mireles-Cabodevila E, Mehta AC, Aboussouan LS. Sudden onset of dyspnea preceded by shoulder and arm pain. Ann Am Thorac Soc. 2016;13(12):2261-2265.

22. Patterson DL, DeRemee RA, Hunt LW. Severe asthma complicated by bilateral diaphragmatic paralysis attributed to Parsonage-Turner syndrome. Mayo Clin Proc. 1994;69(8):774-778.

23. Odell JA, Kennelly K, Stauffer J. Phrenic nerve palsy and ParsonageTurner syndrome. Ann Thorac Surg. 2011;92(1):349-351.

24. Feinberg JH, Radecki J. Parsonage-Turner syndrome. HSS J. 2010;6(2):199-205.

25. Hughes PD, Polkey Ml, Moxham J, Green M. Long-term recovery of diaphragm strength in neuralgic amyotrophy. Eur Respir J. 1999;13(2): 379-384.

26. Golarz SR, White JM. Anatomic variation of the phrenic nerve and brachial plexus encountered during 100 supraclavicular decompressions for neurogenic thoracic outlet syndrome with associated postoperative neurologic complications. Ann Vasc Surg. 2020;62:70-75

27. Loukas M, Kinsella CR Jr, Louis RG Jr, et al. Surgical anatomy of the accessory phrenic nerve. Ann Thorac Surg. 2006;82(5):1870-1875.
28. Graves MJ, Henry BM, Hsieh WC, et al. Origin and prevalence of the accessory phrenic nerve: a meta-analysis and clinical appraisal. Clin Anat. 2017;30(8):1077-1082.

\section{Disclosures}

The authors report no conflict of interest concerning the materials or methods used in this study or the findings specified in this paper.

\section{Author Contributions}

Acquisition of data: Farrell, Weiss. Analysis and interpretation of data: Mathew, Dickerman. Drafting the article: Mathew, Farrell. Critically revising the article: Mathew, Farrell, Dickerman. Reviewed submitted version of manuscript: Mathew, Farrell, Weiss. Approved the final version of the manuscript on behalf of all authors: Mathew.

\section{Supplemental Information}

Previous Presentations

The abstract was presented at the JPS Research Symposium, John Peter Smith Hospital, Fort Worth, Texas, June 5, 2020, and at Research Appreciation Day, University of North Texas Health Science Center, Fort Worth, Texas, March 27, 2020.

\section{Correspondence}

Ezek Mathew: Texas College of Osteopathic Medicine, Fort Worth, TX. ezekmathew@my.unthsc.edu. 